\title{
LIGMA - Was ist das? Wofür ist es gut? Wobei hilft es?
}

\author{
Angelika Kunz, Karin Preiß, Astrid Fuhrmann, Susanne Bäuerle
}

\section{Zusammenfassung}

Das Merkwort LIGMA ist eine „Eselsbrücke“, um sich Fakten und Abläufe leichter zu merken. LIGMA ist aus folgenden Anfangsbuchstaben entstanden:

L - Lagerung

I - Instrumente und Implantate

G - Geräte

$\mathrm{M}$ - Materialien

A - Abläufe

Es soll Schülern und OP-Anfängern helfen, eine OP-Vorbereitung systematisch durchzuführen.

\section{LIGMA - What is it? What's its use? How does it help?}

The acronym LIGMA is a mnemonic in German intended to help memorising facts and processes more easily. LIGMA is made up of the initial letters of the following words:

L - Lagerung (patient positioning)

I - Instrumente und Implantate (instruments and grafts)

G - Geräte (apparatus)

M - Materialien (materials)

A - Abläufe (processes)

It is intended to help students and inexperienced OP personnel to carry out $\mathrm{OP}$ preparation in a systematic manner.

\section{Einleitung}

Um den Schülern der OTA-Ausbildung die Abläufe einzuprägen, „Wie bereite ich eine OP/Osteosynthese vor", kam uns die Idee, ein Merkwort, dessen Anfangsbuchstaben auf eine geordnete Liste von Begriffen verweisen, zu kreieren: „LIGMA“. So eine „Eselsbrücke“ (oder auch „assoziative Arbeitsweise des Gedächtnisses“ genannt), hilft, sich Fakten und Abläufe leichter zu merken.

- L für Lagerung

- I für Instrumente und Implantate

- G für Geräte

- M für Materialien

- A für Abläufe

Es geht um die allgemeine Vorbereitung eines Patienten für eine Operation und nicht nur um ein bestimmtes Osteosyntheseverfahren. tig: Notfall?
OP-JOURNAL 2011; 27: 100-102

(c) Georg Thieme Verlag KG Stuttgart · New York DOI http://dx.doi.org/10.1055/s-0030-1270948 warten bis ans Ende des OP-Tagesprogramms oder sollte er schnellstmöglich im nächsten frei werdenden OPSaal operiert werden?

- Handelt es sich um eine Revision oder gar eine septische Revision?

\section{Welche Information soll das OP-Programm liefern?}

Daraus entsteht die Frage: „Was muss alles vorbereitet werden und welche zusätzlichen Informationen werden noch benötigt?"

- Name, Vorname und Geburtsdatum/ Alter

- Sind Allergien bekannt (z.B. Latex/ Chrom/Nickel)?

- Was soll auf welcher Seite operiert werden?

- Welches Implantat ist geplant?

- Welcher Operateur bzw. Assistent ist für diese OP eingeteilt?

Dabei ist die Information aus dem OPProgramm für die Pflegekraft sehr wich-

- Handelt es sich um eine geplante OP, eine zusätzliche OP oder gar um einen

- Wie dringend sind die zusätzliche OP oder der Notfall? Kann der Patient

- Welche Lagerung wird benötigt (Bauch-, Rücken-, Seiten- oder Beachchair-Lagerung)?

- Welche OP-Zeit ist vorgesehen?

- Welche Art der Narkose ist vorgesehen (ITN, Larynxmaske, Spinal- oder Plexusanästhesie)?

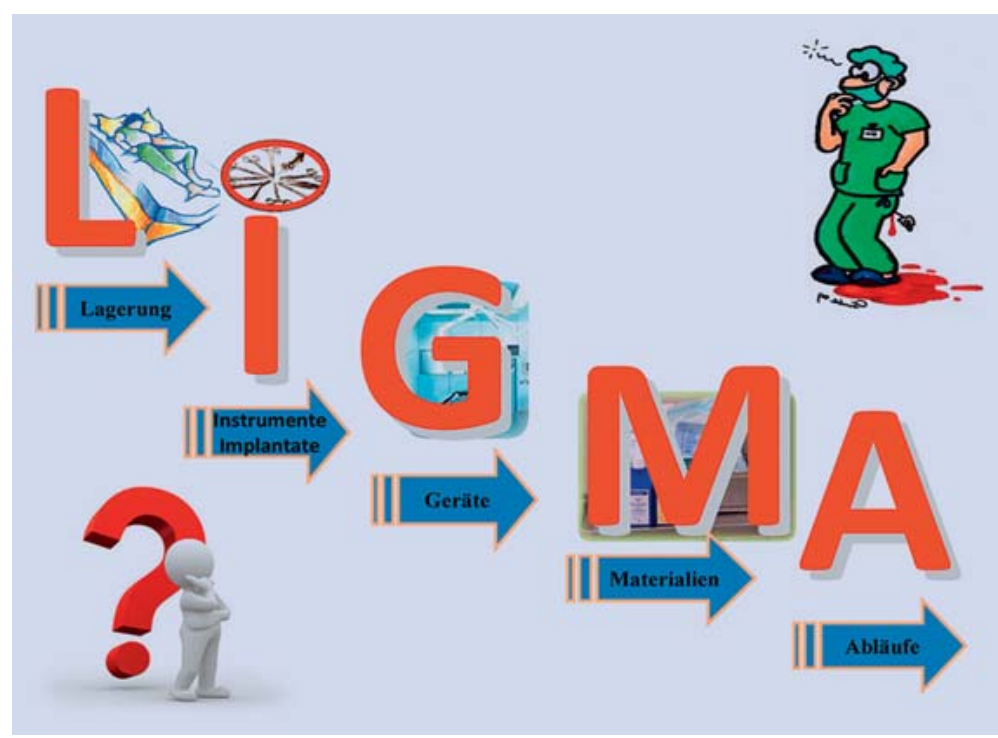


Welche Information soll die Checkliste liefern?

Bevor der Patient an das OP-Team übergeben wird, werden anhand einer Checkliste alle Schwerpunktdaten überprüft und abgeglichen.

- Identität des Patienten, bestenfalls mit Armband

- Geplanter Eingriff und OP-Seite mit Kreuz gekennzeichnet

- Befunde zur OP (Labor, Röntgenbilder, CT oder MRT)

- OP-Aufklärung und Einwilligung für OP und Anästhesie

- Allgemeinzustand des Patienten (Behinderungen, Bewegungseinschränkungen, Schwerhörigkeit)

- Allergien (Latex/Chrom/Nickel)

- Infektbeschreibung (MRSA/HIV/septisch) wichtig für die Patientenübernahme

- Postoperative Hilfsmittel wie Immobilizer, Gilchrist

\section{Wie bereite ich eine OP/Osteosynthese vor}

Damit bei der Vorbereitung für die betreffende Operation nichts Entscheidendes vergessen wird, wird LIGMA als Merkwort angewandt. LIGMA soll eine systematische OP-Vorbereitung fördern.

\section{Lagerung}

- Bevor der Patient in den OP-Saal gebracht wird, muss die Entscheidung über die Lagerung und den benötigten OP-Tisch gefallen sein.

- Soll es ein Universal-, Extensionsoder Wirbelsäulentisch sein, wie und wo muss durchleuchtet werden (zwecks Röntgen-Schutzauflage) oder wird evtl. zur 3-D-Darstellung der Karbontisch benötigt?

- Das Gewicht des Patienten muss beachtet werden. Es könnte ein Schwerlast-OP-Tisch benötigt werden (180$220 \mathrm{~kg}$ ) und es muss abgeklärt werden, ob der betreffende Tischsockel für diese Belastung auch ausgelegt ist.

- Die Vorbereitung des „Lagerungszubehörs“ ist abhängig von der gewünschten Lagerung, ob Extensionszubehör oder Lagerungskissen für eine Bauchlage benötigt werden.

- Mithilfe von Tempur- oder Gelkissen und Matten können Druckstellen oder Nervenschädigungen vermieden und Knochenvorsprünge gepolstert werden.

- Besondere Aufmerksamkeit wird gerichtet auf:
- Kopf

- Schulter und Ellenbogen

- Becken und Hüftknochen

- Knie und Kniekehle

- Innen-, Außenknöchel und Ferse

- Zur Lagerung werden auch wasserundurchlässige Unterlagen benötigt, die nach der Desinfektion wieder entfernt werden, damit der Patient bei der Hautdesinfektion vor Durchfeuchtung geschützt wird und es intraoperativ nicht zu Verbrennungen kommen kann.

\section{Instrumente und Implantate}

- Schon am Vortag wird entsprechend des geplanten OP-Programms eine Fallwagenbestellung aus der Sterilisationsabteilung geordert.

- Die Bestellung soll vorausschauend erfolgen, d. h. einen Osteosynthesewechsel einplanen wie z. B. bei einer trimalleolären OSG-Fraktur. Hier könnte für die Innenknöchelfraktur eine Schrauben- oder Zuggurtungsosteosynthese möglich sein. Bei einer Plattenosteosynthese an langen Röhrenknochen könnte ein Distraktor benötigt werden, je nach Gepflogenheiten des Operateurs.

- Vorausschauendes Überprüfen der Implantate ist wichtig, ob alle Implantate vollständig vorhanden sind oder als Sonderbestellung angefordert werden müssen.

- Abklären, ob spezielle Instrumente zu den Implantaten benötigt werden.

- Sind die nötigen Fachkenntnisse für das Implantationssystem vorhanden für die/den Instrumentierenden? Muss die OP-Anleitung als Abendlektüre studiert werden?

- Zeitnahe Nachbestellung verbrauchter Implantate ist sehr wichtig, ebenso wie die ordnungsgemäße und vollständige Auffüllung der Siebe und der Implantatlager.

\section{Geräte}

- Die Vorbereitung des OP-Saals beginnt mit der Überprüfung der Klimaanlage und der Saalbeleuchtung.

- HF-Geräte einsatzbereit schalten, d.h. die richtige OP-übliche Einstellung überprüfen und ausreichend Neutralelektroden für das OP-Programm bereitlegen.

- Druckluftschlauch an Wandanschluss bzw. Deckenampel anbringen oder geladene Akkus bereitstellen.
- Die Absaugvorrichtung einsatzbereit stellen und eine Funktionskontrolle durchführen.

- Die Blutsperre bereitstellen und anschließen.

- Den C-Bogen für die intraoperative Röntgenkontrolle oder die 3-D-Aufnahmen vorbereiten; mit Namen des Patienten versehen und für das radiologische Informationssystem (RIS) anmelden bzw. Röntgen- und Magnetresonanztomografie-(MRT-)Befundwiedergabe ermöglichen.

- Computer-OP-Dokumentation und Patienteneingaben vervollständigen.

- Abhängig von der geplanten OP werden noch Arthroskopiezubehör wie Shaver, Bildprinter und Monitor oder CD- und Fotodokumentation benötigt.

\section{Materialien}

- Dazu gehören die benötigten OP-Abdeckungen je nach OP-Zugang, als Extremitäten-, Hüft- oder Universalset.

- Bei Verwendung einer Blutsperre werden Manschette und Polsterung benötigt.

- Spüllösungen, Absaugung, Jet-Lavage.

- Bedarfsweise Abstriche für die Mikrobiologie oder Gefäße für histologische Präparate bereitstellen.

- Handschuhe für das OP-Team, Nahtmaterial, Skalpellklingen und Drainagen.

- Sterilabdeckung für den C-Bogen und/ oder die Röntgen-Kassettenbezüge.

- Postoperative Wundabdeckung wie Wundverband oder Druckverbände.

- Rechtzeitiges Bereitstellen von Gips, Cellacast oder Winkler-Schiene, Immobilizer, Gilchrist oder Schanzkrawatte in entsprechenden Größen.

\section{Abläufe}

- Wo und welche Seite soll operiert werden?

- Ist es ein aseptischer oder ein septischer OP-Verlauf?

- Welcher operative Zugang oder welche Zugänge könnten gemacht werden?

- Ist es eine OP mit oder ohne Arthroskopie oder ist eine Spongiosaentnahme geplant?

- Wird eine Blutsperre benötigt?

- Soll eine beidseitige Abdeckung verwendet werden, wie z.B. bei Achillessehnenruptur, Lappenplastik, Spalthautentnahme, Gefäßrekonstruktion oder einzeitiger Versorgung eines polytraumatisierten Patienten? 
- Wird eine intraoperative Umlagerung notwendig oder eine 3-D-Darstellung?

- Welche Art der Narkose ist vorgesehen?

- Welches Vorgehen ist bei ArmplexusAnästhesie geplant für die Entnahme von Spongiosa aus dem Beckenkamm?

- Wie lange ist die voraussichtliche OPZeit?

- Ist das Legen eines Blasenkatheters notwendig?

- Organisation von fachübergreifender Versorgung eines Patienten sollte rechtzeitig geplant werden.

Mit dem Merkwort und der Umsetzung von LIGMA kann die Einarbeitungsphase von Schülern und auch neuen Mitarbeitern optimiert werden.

Der Start ins Berufsleben als OP-Pflegekraft kann somit erleichtert werden.

\section{Angelika Kunz}

OP-Schwester

Klinik und Poliklinik für Unfall- und

Wiederherstellungschirurgie

Universitätsklinikum

C.G. Carus Dresden

Fetscherstraße 74

01307 Dresden

angelika.kunz@uniklinikum-

dresden.de

Karin Preiß

OP-Schwester

Klinik und Poliklinik

Viszeral-Thorax- und Gefäßchirurgie

Universitätsklinikum

C.G. Carus Dresden

Fetscherstraße 74

01307 Dresden

\section{Astrid Fuhrmann}

Diplom-Medizinpädagogin

Medizinische Berufsfachschule

Krankenhaus Dresden Friedrichstadt

Friedrichstraße 41

01067 Dresden

\section{Susanne Bäuerle}

Senior Project Manager ORP

\section{AO Trauma}

Clavadelerstrasse 8

7270 Davos

Schweiz 